\title{
BMJ Open Comparative efficacy and acceptability of antiepileptic drugs for classical trigeminal neuralgia: a Bayesian network meta-analysis protocol
}

\author{
Zongshi Qin, ${ }^{1,2}$ Shang Xie, ${ }^{3}$ Zhi Mao, ${ }^{4}$ Yan Liu, ${ }^{5}$ Jiani Wu, ${ }^{1}$ Toshi A. Furukawa, ${ }^{6}$ \\ Joey S.W. Kwong, ${ }^{7}$ Jinhui Tian, ${ }^{8}$ Zhishun Liu ${ }^{1}$
}

To cite: Qin Z, Xie S, Mao Z, et al. Comparative efficacy and acceptability of antiepileptic drugs for classical trigeminal neuralgia: a Bayesian network metaanalysis protocol. BMJ Open 2018;8:e017392. doi:10.1136/ bmjopen-2017-017392

- Prepublication history and additional material for this paper are available online. To view these files, please visit the journal online (http://dx.doi. org/10.1136/bmjopen-2017017392).

Received 20 April 2017 Revised 24 October 2017 Accepted 8 November 2017

Check for updates

For numbered affiliations see end of article.

Correspondence to Professor Zhishun Liu; liuzhishun@aliyun.com

\section{ABSTRACT}

Introduction Trigeminal neuralgia (TN) affects 4 to 28.9/100 000 people worldwide, and antiepileptic drugs such as carbamazepine and oxcarbazepine are the firstline treatment options. However, the efficacy and safety of other antiepileptic drugs remain unclear due to insufficient direct comparisons.

Objective To compare the efficacy and acceptability of all currently available antiepileptic agents for the treatment of patients with classical TN.

Methods We will search the PubMed, EMBASE, Cochrane Library and Web of Science databases for unpublished or undergoing research listed in registry platforms. We will include all randomised controlled trials comparing two different antiepileptic drugs or one antiepileptic drug with placebo in patients with classical TN. The primary outcomes will be the proportion of responders and the number of subjects who dropout during the treatment. The secondary outcomes will include the two primary outcomes but in the follow-up period, changes in the selfreporting assessment scale for neuralgia and quality of life assessment. In terms of network meta-analysis, we will fit our model to a Bayesian framework using the JAGS and pcnetmeta packages of the $\mathrm{R}$ project.

Ethics and dissemination This protocol will not disseminate any private patient data. The results of this review will be disseminated through peer reviewed publication.

PROSPERO registration number CRD42016048640.

\section{INTRODUCTION}

Classical trigeminal neuralgia $(\mathrm{TN}), \mathrm{a}$ chronic pain disorder described as one of the most severe pains one can suffer, is characterised by paroxysms of unilateral, electric shock-like severe pain along the trigeminal nerve divisions. ${ }^{12}$ It affects lifestyle because it can be triggered by common activities, such as eating, talking, shaving or brushing your teeth. The wind, chewing and talking also aggravate the condition in many patients. ${ }^{2}$ It is estimated that approximately 4 to 28.9 per 100000 people worldwide suffer from TN, and the number affected tends to be higher

\section{Strengths and limitations of this study}

To the best of the authors' knowledge, this study will be the first network meta-analysis to assess the comparative efficacy and acceptability of all the available antiepileptic drugs for the treatment of classical trigeminal neuralgia.

- This study will be performed by Bayesian framework, which will enable us to estimate the probability of each intervention to be the best for each outcome.

- Owing to language barriers, the number of included trials may be potentially limited.

among women at all ages and even increases with age. ${ }^{34}$

At present, the cause of $\mathrm{TN}$ remains unclear. ${ }^{56}$ One hypotheses is that the trigeminal nerve becomes compressed at the root entry zone by cerebral vessels. ${ }^{7}$ Owing to the contradictory aetiology and poorly understood pathophysiological mechanisms underlying $\mathrm{TN}$, a variety of therapeutic and surgical approaches have been developed to alleviate the associated pain and improve the quality of life for patients with classical TN. ${ }^{8-10}$ Although many patients have obtained excellent outcomes from surgery, many others do not experience any pain relief. ${ }^{11} 12$ Furthermore, the currently available surgical procedures are associated with various complications, particularly sensory loss in the trigeminal nerve territory, anaesthesia dolorosa and, rarely, ipsilateral hearing loss, depending on the technique. ${ }^{13} 14$

Hence pharmacological measures to improve clinical outcomes are needed. The most commonly used option is antiepileptic drugs, with phenytoin being the first drug to be used for classical $\mathrm{TN}$ with a positive effect. ${ }^{15}$ Carbamazepine can reduce both the frequency and intensity of painful paroxysms and was first introduced by the US Food and 
Drug Administration; however, its efficacy is compromised by poor tolerability. ${ }^{16}$ Oxcarbazepine, a derivative of carbamazepine, is often used as an initial treatment for classical TN and has more favourable properties than carbamazepine related to its increased efficacy in epilepsy, greater tolerability and decreased potential for drug interactions. ${ }^{17}$ Lamotrigine has also been reported as an effective add-on therapy, ${ }^{18}$ whereas there is little evidence that other antiepileptic drugs, such as clonazepam, gabapentin, pregabalin and valproate, have a beneficial effect. ${ }^{19-22}$ However, many of the studies are old with limited methodology, and were assessed as having low Grading of Recommendations Assessment, Development and Evaluation (GRADE) scores. ${ }^{23}$

To date, several systematic reviews have investigated the comparative efficacy and safety of antiepileptic drugs. ${ }^{20}{ }^{24-28}$ However, previous systematic reviews have only considered pairwise evidence from head to head comparisons and have thus failed to assess the comparative efficacy and acceptability of all the available antiepileptic drugs. Thus it is difficult to determine the best treatments for relieving pain with minimal adverse effects. In the present study, we choose a group of nine antiepileptic drugs, looking at drugs which were licensed for neuralgia in many countries and which were frequently used in clinical practice. We will apply network meta-analysis to integrate direct and indirect comparisons, ${ }^{29} 30$ which could be used not only to strengthen inferences concerning the efficacy and acceptability of treatments but also to rank the efficacy and acceptability of antiepileptic drugs accordingly. ${ }^{31}$

The objectives of this systematic review and network meta-analysis are: (1) to compare all currently available antiepileptic drugs in terms of efficacy and acceptability in the treatment of classical TN; and (2) to determine which drug achieves the best balance between efficacy and adverse effects. The results of this study will augment findings based on current pairwise meta-analyses and are expected to provide important information to support clinical practice and health policy decisions.

\section{METHODS}

This protocol will be conducted in accordance with the reporting guidance provided in the Preferred Reporting Items for Systematic Reviews and Meta-Analysis Protocols (PRISMA-P) statement and Checklist of Items to Include When Reporting a Systematic Review Involving a Network Meta-analysis. ${ }^{32} 33$ The protocol is registered in PROSPERO (CRD: 42016048640). This study will not involve any private patient data; ethics approval was waived (see online supplementary file 1 for PRISMA-P checklist).

\section{Eligibility criteria}

Study types

We will include randomised controlled trials (RCTs) comparing one antiepileptic drug with another antiepileptic drug as monotherapy or placebo for the treatment of TN. Quasi-randomised controlled trails allocating participants according to birth date or the consequences of enrolment will be excluded. The minimum duration for RCT inclusion will be set at 4 weeks. Trials with more than a two arm design will be considered only if the available data meet the criteria for an intervention. For trials with a crossover design, data will only be extracted from the first randomisation period.

\section{Participant characteristics}

Only trials that enrolled participants with a diagnosis of classical TN according to standardised criteria, such as the International Headache Society's classification, International Classification of Headache Disorders, will be sought. ${ }^{134}$ For studies using other extensive criteria for the diagnosis of classical TN, detailed diagnostic criteria must be reported (such as history or characteristics that have been confirmed by CT or MRI) ${ }^{35}$ Studies examining symptomatic TN patients will not be included. Participants with comorbid conditions, such as anxiety, depression, epilepsy or other medical conditions, will not be eligible for inclusion. No limitations will be imposed on age, sex or nationality.

\section{Intervention types}

We plan to include the following antiepileptic drugs: carbamazepine, lamotrigine, clonazepam, phenytoin, valproate, gabapentin, pregabalin, oxcarbazepine and topiramate. In addition to these antiepileptic drugs, we will also obtain information about interventions of interest from either pairwise RCTs or placebo controlled trails, as some RCTs design a placebo controlled arm as the comparator. Figure 1 illustrates the network plot of all possible direct comparisons between the eligible interventions.

\section{Outcome measures}

Studies reporting one of the following will be included.

\section{Primary outcomes}

The primary objective of this review is to assess the efficacy and acceptability of antiepileptic drugs for classical $\mathrm{TN}$; therefore, the following two outcomes will be used as the primary outcomes.

1. The proportion of responders to a self-reporting assessment scale for neuralgia. A responder was defined as a subject who obtained $\geq 50 \%$ reduction in pain score from baseline to the study endpoint (4-12 weeks) or a subject who obtained a pain reducing score of no less than the minimal clinically important difference. Pain scores will be extracted based on the visual analogue score, numerical rating score or any other validated scale for the assessment of overall TN symptoms when available. ${ }^{36}$

2. Treatment acceptability is defined as the proportion of patients who have intervention related adverse events during the 4-12 weeks. 


\section{Secondary outcomes}

1. The proportion of responders with $\geq 50 \%$ pain reduction on a self-reporting assessment scale for neuralgia from baseline to the endpoint after follow-up.

2. The change in pain symptoms of TN from baseline to the endpoint (4-12 weeks), based on the visual analogue score, numerical rating score or any other validated scale for the assessment of overall TN symptoms when available.

3. The change in pain symptoms of $\mathrm{TN}$ from baseline to the endpoint after follow-up.

4. The quality of life based on measurement with a validated scale, such as the Short Form 36 Health Survey questionnaire. $^{37}$

\section{Search strategy}

We will identify RCTs through a comprehensive, systematic literature search, primarily utilising the PubMed, EMBASE, Cochrane Library and Web of Science databases. As publication bias caused by insufficient unpublished data can significantly bias the comparative efficacy results of network meta-analyses and modify rankings, we will also perform searches for unpublished or ongoing trials using the System for information on Grey Literature in Europe (SIGLE) as well as other registry platforms, such as Clinicaltrials.gov and the International Clinical Trials Registry Platform. Prior to completing this review, we will perform an additional search of each database and registration platform to guarantee that the most recent studies are included. We will use medical subject headings and text words related to 'trigeminal neuralgia' and 'randomised controlled trial' for the literature search. In addition, the reference lists of previous systematic reviews will be examined to ensure the quantity and accuracy of the included studies. The search strategy will be developed by JT and ZL; we anticipate that the databases will be searched from their inception to 30 September 2017 (see online supplementary file 2 for the search strategies for PubMed, EMBASE and Cochrane Library).

\section{Data collection process}

Two authors (SX and ZM) will scan the titles and abstracts of the trials after duplicated records have been excluded using EndNote X7 (Thomson Reuters, New York, New York, USA). The scanning will be performed using EndNote, and all trials will be allocated to the following five groups: inclusion group, non-patient group, intervention group, outcome group and awaiting group. A prior data collection process will be conducted using an electro table created with Excel software, which has been used in our previous study. ${ }^{38}$ The table will consist of four sheets, including general information (author list, publication year and journal), characteristics of the included trials (diagnostic criteria, age range, study drugs and dose range), risk of bias assessed using the Cochrane risk of bias tool and outcome data extraction (number of participants who responded to treatment and the number who dropped out during the treatment). All original data will be submitted as an attachment. A flowchart illustrating this design is presented in figure 2 .

\section{Quality assessment}

Two authors (JW and YL) will use the Cochrane risk of bias tool to assess the risk of bias of the eligible studies,

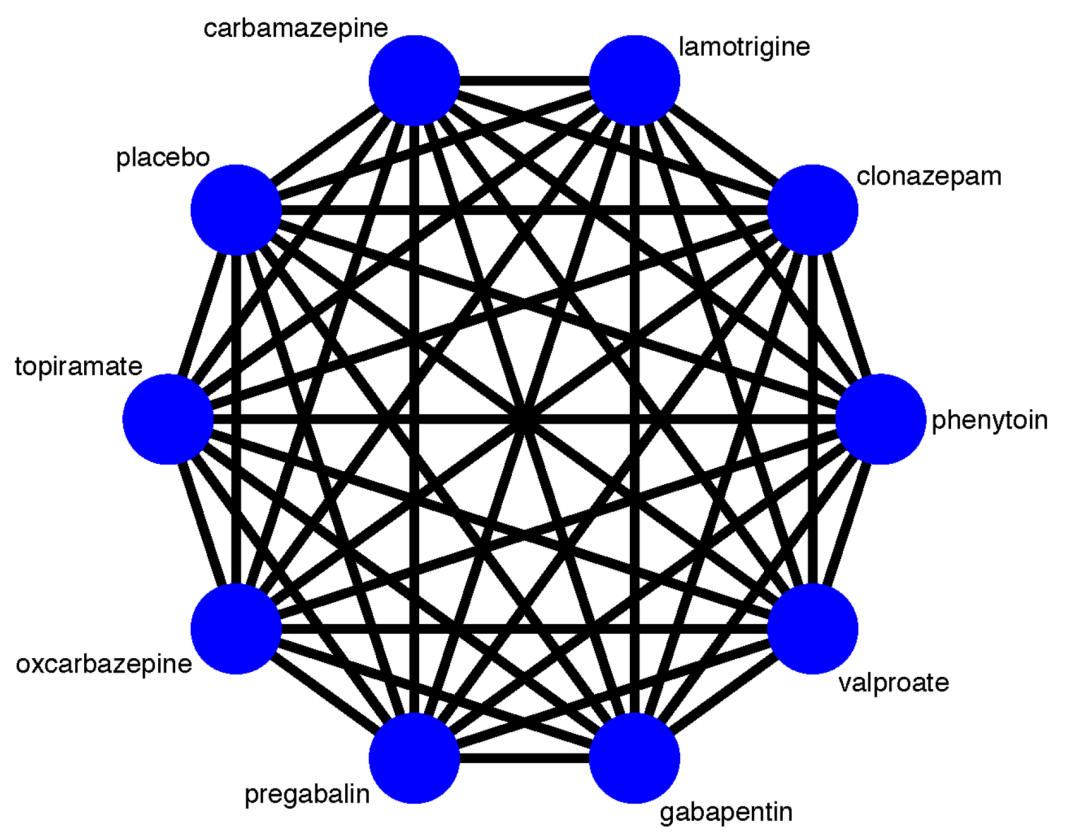

Figure 1 Network plot of all possible direct comparisons between the eligible interventions. 


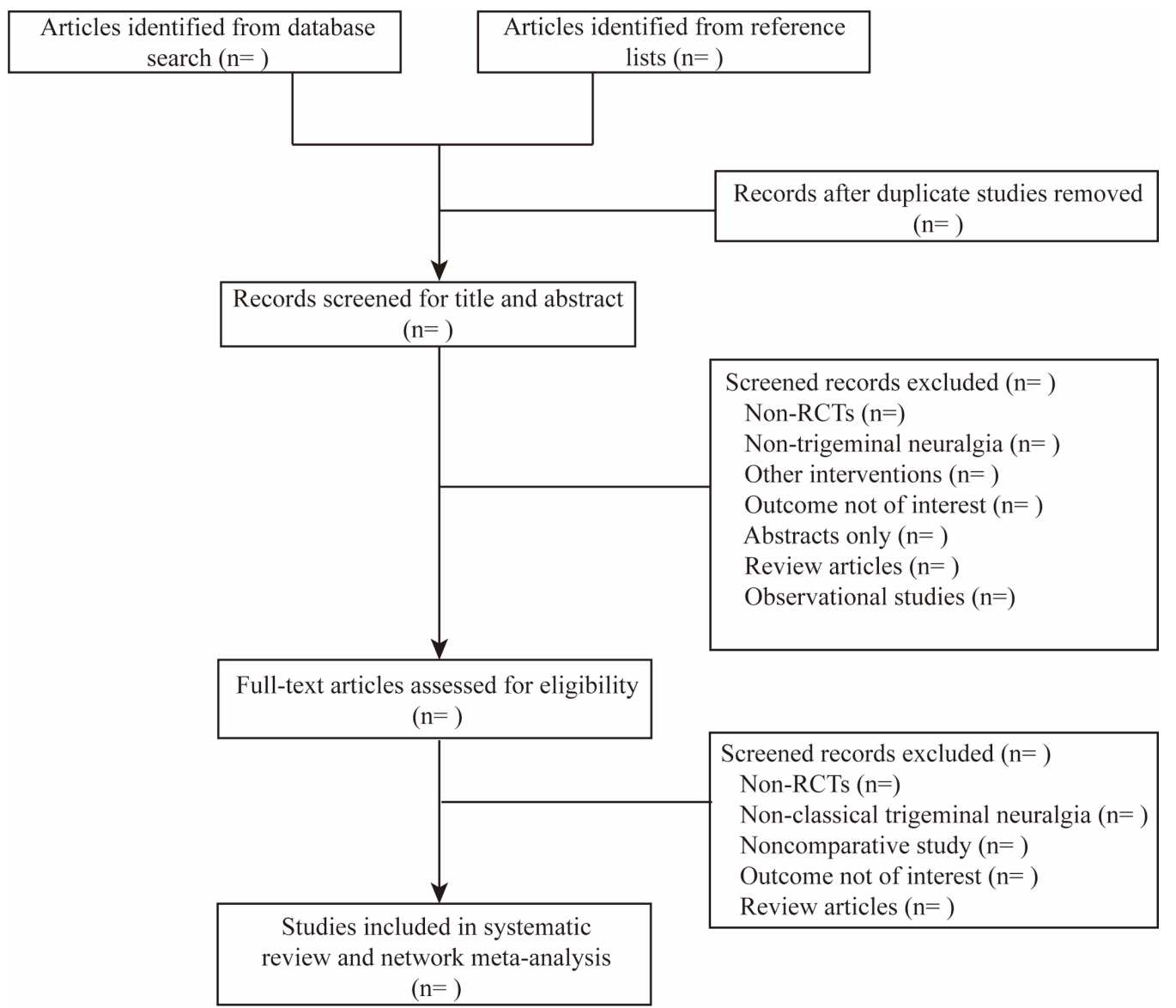

Figure 2 PRISMA flowchart. RCT, randomised controlled trial.

covering randomisation, concealment allocation, blinding and other biases. ${ }^{39}$ As inadequate concealment could potentially fail the randomisation test, two independent review authors will pay particular attention to the adequacy of random allocation concealment and blinding. The other sources of bias will be assessed considering the sample size calculation method, diagnostic criteria, reporting of withdrawals and follow-up. Two authors (JSWK and JT) will assess the quality of evidence using the GRADE framework, covering study limitations, inconsistency, indirectness, imprecision and publication bias. ${ }^{40}$ The methods for rating the quality of direct comparisons are the same as the methods used in traditional meta-analyses, and the following steps will be used in the whole assessment procedure: (1) presenting direct and indirect effect estimates; (2) rating the quality of direct and indirect estimates; (3) presenting the results of the network meta-analysis; and (4) rating the quality of the network meta-analysis effect estimates.

\section{Dealing with missing data}

To obtain missing data, we will initially contact the senior or corresponding author. If no one responds, we will estimate the missing data as follows. For studies failing to report the number of responding patients after treatment, instead of providing the mean and SD, we will calculate the number of responding patients employing a validated imputation method. ${ }^{41}$ In addition, we will also estimate missing data from graphs when possible. For trials that cannot be extracted or estimated, the available data will be excluded, and the reason for exclusion will be reported.

\section{Statistical analysis}

The method used for data synthesis will be based on mixed treatment meta-analysis. To examine comparisons, we will use Stata (13.0; Stata Corporation, College Station, Texas, USA) to synthesise data and will present the comparison results if the included studies are sufficient for each pairwise comparison. We will use a random effects model to combine the data, and the outcomes of continuous and binary variables will be presented as standardised mean differences (SMDs) and ORs with 95\% CIs. For indirect comparisons, we will perform an arm based network meta-analysis for all treatments using a random effects model with a Bayesian framework using the pcnetmeta package of the $\mathrm{R}$ project, which can conduct calculations using JAGS software. ${ }^{42-44}$ This will enable us to estimate the best probability of each intervention for each positive outcome, given the results of the multiple treatment meta-analysis. At least one network focusing on the response rate for pain relief will be constructed, in which a statistically significant difference defined as the null value will not 
be included in the $95 \%$ CI. All models will be utilised for 50000 simultaneous iterations based on the data and the description of the proposed distributions for relevant parameters, and the first 10000 iterations will be discarded to avoid potential impact on the arbitrary value. For continuous outcomes and binary outcomes, the OR and SMD values will be presented with the $95 \%$ credible interval ( $\mathrm{CrI})$.

To describe relationships among different treatments, a network plot will be created to show direct comparisons between arms based on different outcomes. ${ }^{42}$ In addition, the effectiveness of each treatment among all available treatments will be ranked by calculating the OR in order, and plots of the treatment rank probabilities will be generated to rank the various treatments for each outcome using the functions in package pcnetmeta. ${ }^{42}{ }^{43}$ We will also present a cluster rank table to synthesise the efficacy and acceptability of each drug (using two primary outcomes). The table will consist of two triangles: the upper right triangle will illustrate the acceptability and the lower left triangle will illustrate the efficacy. ${ }^{31}$ For pairwise meta-analyses we will use Stata 13.0. For network meta-analyses we will use JAGS and $\mathrm{R}$ project.

\section{Assessment of heterogeneity}

Heterogeneity, which plays a pivotal role in both standard meta-analyses and network meta-analyses, refers to the degree of disagreement between study specific treatment effects and constitutes the basis of inconsistency. To test the heterogeneity of each pairwise comparison, we will use the $\mathrm{I}^{2}$ statistic. ${ }^{45}$

\section{Assessment of transitivity and similarity}

In addition to the heterogeneity assessment using the $\mathrm{I}^{2}$ statistic, the assumption of transitivity and similarity based on clinical and methodological characteristics will be assessed. It should be noted that it is difficult to identify these effect modifiers using statistical analysis. We will assume that intervention effects are transitive in this network meta-analysis because we will only focus on antiepileptic drugs, and we will investigate similarity based on clinical characteristics, such as antiepileptic drug dose, period of treatment and severity of pain symptoms at baseline, as well as according to methodological characteristics, such as study quality. ${ }^{46}$ All of these effect modifiers will be judged and reported before the network meta-analysis is conducted.

\section{Assessment of inconsistency}

Evaluation and explanation of inconsistency is another basic objective of a network meta-analysis. In this context, inconsistency refers to the degree of difference between direct and indirect comparisons and can be evaluated only when a loop exists in the evidence network. This means that inconsistency assessment using a design by treatment interaction model cannot be conducted if the structure of this network is a 'star network' (ie, all interventions have a single mutual comparator, such as a placebo).${ }^{47}$ For such cases, we will test inconsistency using a node splitting model. ${ }^{49}$

To identify inconsistency among the included trials of the network, we will use Stata, performing the $\mathrm{Z}$ test to compare direct and indirect summary effects in specific loops. ${ }^{50}$ If there is no inconsistency between loops or designs, we will use a consistency model to calculate the data. For cases of significant incoherence, we will initially look for data extraction errors in loops that present inconsistency and in comparisons with large heterogeneity. ${ }^{51}$ After the data have been scrutinised, we will investigate possible sources of inconsistency within the clinical and methodological variables suspected of being potential sources of either heterogeneity or incoherence in each comparison specific group of trials. If an important inconsistency cannot be explained, we will consider avoiding synthesis of the related network.

\section{Additional analyses}

To ensure the quality of this review, studies not reporting blinding will be excluded prior to data synthesis because blinding plays a vital important role in the RCT. We will assess heterogeneity quantitatively using the $\mathrm{I}^{2}$ statistic, and if an $\mathrm{I}^{2}$ value is $>50 \%$, we will explore the source of heterogeneity. We will initially perform sensitivity analysis by excluding trials rated as having a high risk of bias. Additionally, meta-regression or subgroup analysis will be used to explore possible sources of heterogeneity if the number of included trials is sufficient. For network meta-regression, we will use a random effects network meta-regression model to examine potential factors.

\section{DISCUSSION}

To the best of our knowledge, no network meta-analyses comparing the use of antiepileptic drugs for the treatment of classical TN have been conducted to date. Previous systematic reviews have compared only a single drug to other types of drug or therapy. ${ }^{2024-28}$ This makes it difficult to obtain a clear understanding of the effectiveness of the various different conservative treatments for this disorder. A network meta-analysis can be used to perform indirect comparisons and allows parameters for direct and indirect comparisons to be synthesised. To ensure the quantity and quality of the potentially included RCTs, we will perform an extensive literature search and predefine rigorous inclusion criteria. Also, we will assess the quality of evidence using the GRADE framework. Although a ranking of the included interventions will be generated, with the exception of findings, the quality of evidence should also be considered. We hope that the results of this review will help clinicians make more accurate treatment decisions and promote additional research into conservative treatments for classical TN. 


\section{Amendments}

If it is necessary we will update this protocol in the future. We will submit the original protocol, final protocol and summary of changes as a supplement.

\section{Author affiliations}

${ }^{1}$ Department of Acupuncture and Neurology, Guang'anmen Hospital, China Academy of Chinese Medical Sciences, Beijing, China

${ }^{2}$ School of Life Sciences, Beijing University of Chinese Medicine, Beijing, China ${ }^{3}$ Department of Oral and Maxillofacial Surgery, Peking University School and Hospital of Stomatology, Beijing, China

${ }^{4}$ Department of Critical Care Medicine, Chinese People's Liberation Army General Hospital, Beijing, China

${ }^{5}$ Data Centre of Traditional Chinese Medicine, China Academy of Chinese Medical Sciences, Beijing, China

${ }^{6}$ Department of Health Promotion and Human Behaviour, Kyoto University Graduate School of Medicine/School of Public Health, Kyoto, Japan

${ }^{7} \mathrm{JC}$ School of Public Health and Primary Care, Faculty of Medicine, The Chinese University of Hong Kong, Hong Kong, Hong Kong, China

${ }^{8}$ Evidence Based Medicine Centre, Lanzhou University, Lanzhou, China

Contributors ZQ, SX and ZM conceived the study, and contributed equally to this study. JT and SX developed the search strategies. ZQ and SX wrote the first draft. TAF and ZL revised the draft. SX and ZM will independently screen potential studies and extract data from the included studies. JW, JSWK, JT and YL will assess the risk of bias and summarise the evidence. ZM, SX, JSWK and ZL will address the missing data, if any. ZQ and JT will perform the statistical analysis. ZL and JT will arbitrate in cases of disagreement and ensure the absence of errors. All authors approve the publication of this protocol.

Funding This research received no specific grant from any funding agency in the public, commercial or not-for-profit sectors.

Competing interests None declared.

Patient consent Parental/guardian consent obtained.

Provenance and peer review Not commissioned; externally peer reviewed.

Open Access This is an Open Access article distributed in accordance with the terms of the Creative Commons Attribution (CC BY 4.0) license, which permits others to distribute, remix, adapt and build upon this work, for commercial use, provided the original work is properly cited. See: http://creativecommons.org/ licenses/by/4.0/

(c) Article author(s) (or their employer(s) unless otherwise stated in the text of the article) 2018. All rights reserved. No commercial use is permitted unless otherwise expressly granted.

\section{REFERENCES}

1. Headache Classification Committee of the International Headache Society (IHS). The international classification of headache disorders, 3rd edition (beta version). Cephalalgia 2013;33:629-808

2. Zakrzewska JM, Linskey ME. Trigeminal neuralgia. BMJ 2015;350:h1238.

3. Mueller D, Obermann M, Yoon MS, et al. Prevalence of trigeminal neuralgia and persistent idiopathic facial pain: a population-based study. Cephalalgia 2011;31:1542-8.

4. MacDonald BK, Cockerell OC, Sander JW, et al. The incidence and lifetime prevalence of neurological disorders in a prospective community-based study in the UK. Brain 2000;123:665-76.

5. Devor M, Amir R, Rappaport ZH. Pathophysiology of trigeminal neuralgia: the ignition hypothesis. Clin J Pain 2002;18:4-13.

6. DeSouza DD, Hodaie M, Davis KD. Structural magnetic resonance imaging can identify trigeminal system abnormalities in classical trigeminal neuralgia. Front Neuroanat 2016;10:95.

7. Katusic S, Beard CM, Bergstralh E, et al. Incidence and clinical features of trigeminal neuralgia, Rochester, Minnesota, 1945-1984. Ann Neurol 1990;27:89-95.

8. Gronseth G, Cruccu G, Alksne J, et al. Practice parameter: the diagnostic evaluation and treatment of trigeminal neuralgia (an evidence-based review): report of the quality standards subcommittee of the American Academy of Neurology and the European Federation of Neurological Societies. Neurology 2008;71:1183-90.

9. Campbell FG, Graham JG, Zilkha KJ. Clinical trial of carbazepine (tegretol) in trigeminal neuralgia. J Neurol Neurosurg Psychiatry 1966;29:265-7.

10. Fromm GH, Terrence CF, Chattha AS. Baclofen in the treatment of trigeminal neuralgia: double-blind study and long-term follow-up. Ann Neurol 1984;15:240-4.

11. Zakrzewska JM, Lopez BC, Kim SE, et al. Patient reports of satisfaction after microvascular decompression and partial sensory rhizotomy for trigeminal neuralgia. Neurosurgery 2005;56:1304-12.

12. Nanda A, Javalkar V, Zhang S, et al. Long term efficacy and patient satisfaction of microvascular decompression and gamma knife radiosurgery for trigeminal neuralgia. $J$ Clin Neurosci 2015;22:818-22.

13. Linskey ME, Ratanatharathorn V, Peñagaricano J. A prospective cohort study of microvascular decompression and Gamma Knife surgery in patients with trigeminal neuralgia. J Neurosurg 2008;109:160-72.

14. Zakrzewska JM, Thomas DG. Patient's assessment of outcome after three surgical procedures for the management of trigeminal neuralgia. Acta Neurochir 1993;122:225-30.

15. Sindrup $\mathrm{SH}$, Jensen TS. Pharmacotherapy of trigeminal neuralgia. Clin J Pain 2002:18:22-7.

16. Wiffen PJ, McQuay HJ, Moore RA. Carbamazepine for acute and chronic pain. Cochrane Database Syst Rev 2005;3:CD005451.

17. Zakrzewska JM, Patsalos PN. Long-term cohort study comparing medical (oxcarbazepine) and surgical management of intractable trigeminal neuralgia. Pain 2002;95:259-66.

18. Zakrzewska JM, Chaudhry Z, Nurmikko TJ, et al. Lamotrigine (lamictal) in refractory trigeminal neuralgia: results from a doubleblind placebo controlled crossover trial. Pain 1997;73:223-30.

19. Court JE, Kase CS. Treatment of tic douloureux with a new anticonvulsant (clonazepam). J Neurol Neurosurg Psychiatry 1976;39:297-9.

20. Yuan M, Zhou HY, Xiao ZL, et al. Efficacy and safety of gabapentin vs. carbamazepine in the treatment of trigeminal neuralgia: a metaanalysis. Pain Pract 2016;16:1083-91.

21. Obermann M, Yoon MS, Sensen K, et al. Efficacy of pregabalin in the treatment of trigeminal neuralgia. Cephalalgia 2008;28:174-81.

22. Peiris JB, Perera GL, Devendra SV, et al. Sodium valproate in trigeminal neuralgia. Med J Aust 1980;2:278.

23. Zakrzewska JM, Linskey ME. Trigeminal neuralgia. Clin Evid 2014;10:1207.

24. McQuay H, Carroll D, Jadad AR, et al. Anticonvulsant drugs for management of pain: a systematic review. BMJ 1995;311:1047-52.

25. Wiffen PJ, Rees J. Lamotrigine for acute and chronic pain. Cochrane Database Syst Rev 2007;2:CD006044.

26. Wiffen PJ, McQuay HJ, Rees J, et al. Gabapentin for acute and chronic pain. Cochrane Database Syst Rev 2005;3:CD005452.

27. Wiffen PJ, Derry S, Moore RA, et al. Carbamazepine for acute and chronic pain in adults. Cochrane Database Syst Rev 2011;1:CD005451.

28. Moore RA, Straube S, Wiffen PJ, et al. Pregabalin for acute and chronic pain in adults. Cochrane Database Syst Rev 2009;3:CD007076.

29. Caldwell DM, Ades AE, Higgins JP. Simultaneous comparison of multiple treatments: combining direct and indirect evidence. BMJ 2005;331:897-900.

30. Cipriani A, Higgins JP, Geddes JR, et al. Conceptual and technical challenges in network meta-analysis. Ann Intern Med 2013;159:130-7.

31. Cipriani A, Furukawa TA, Salanti G, et al. Comparative efficacy and acceptability of 12 new-generation antidepressants: a multipletreatments meta-analysis. Lancet 2009;373:746-58.

32. Shamseer L, Moher D, Clarke M, et al. Preferred reporting items for systematic review and meta-analysis protocols (PRISMA-P) 2015: elaboration and explanation. BMJ 2015;349:97647.

33. Hutton B, Salanti G, Caldwell DM, et al. The PRISMA extension statement for reporting of systematic reviews incorporating network meta-analyses of health care interventions: checklist and explanations. Ann Intern Med 2015;162:777-84.

34. Merskey H, Bogduk N. Classification of chronic pain: Descriptions of chronic pain syndromes and definitions of pain terms. Seattle, USA: IASP Press, 1994:59-71.

35. Zakrzewska JM. Diagnosis and differential diagnosis of trigeminal neuralgia. Clin J Pain 2002;18:14-21.

36. Dworkin RH, Turk DC, Farrar JT, et al. Core outcome measures for chronic pain clinical trials: IMMPACT recommendations. Pain 2005;113:9-19. 
37. Ware JE, Sherbourne CD. The MOS 36-item short-form health survey (SF-36). I. Conceptual framework and item selection. Med Care 1992;30:473-83.

38. Qin Z, Li B, Wu J, et al. Acupuncture for chronic diarrhea in adults: protocol for a systematic review. Medicine 2017;96:e5952.

39. Higgins JPT, Altman DG, Sterne JAC. Chapter 8: Assessing risk of bias in included studies. In: Higgins JPT, Green S, eds. Cochrane Handbook for Systematic Reviews of Intervention, version 5.1.0 (updated March 2011): The Cochrane Collaboration, 2011. Available from www.cochrane-handbook.org

40. Salanti G, Del Giovane C, Chaimani A, et al. Evaluating the quality of evidence from a network meta-analysis. PLoS One 2014;9:e99682.

41. Furukawa TA, Cipriani A, Barbui C, et al. Imputing response rates from means and standard deviations in meta-analyses. Int Clin Psychopharmacol 2005;20:49-52.

42. Lin L, Zhang J, Hodges JS, et al. Performing arm-based network meta-analysis in R with the pcnetmeta package. J Stat Softw $2017 ; 80$.

43. Lin L, Zhang J, Chu H. pcnetmeta: methods for patient-centered network meta-analysis: R package version 2.4, 2016. http://CRAN. R-project.org/package=pcnetmeta
44. Zhang J, Carlin BP, Neaton JD, et al. Network meta-analysis of randomized clinical trials: reporting the proper summaries. Clin Trials 2014;11:246-62.

45. Higgins JP, Thompson SG, Deeks JJ, et al. Measuring inconsistency in meta-analyses. BMJ 2003;327:557-60.

46. Baker SG, Kramer BS. The transitive fallacy for randomized trials: if a bests $b$ and $b$ bests $c$ in separate trials, is a better than $c$ ? BMC Med Res Methodol 2002;2:13.

47. White IR, Barrett JK, Jackson D, et al. Consistency and inconsistency in network meta-analysis: model estimation using multivariate metaregression. Res Synth Methods 2012;3:111-25.

48. Higgins JP, Jackson D, Barrett JK, et al. Consistency and inconsistency in network meta-analysis: concepts and models for multi-arm studies. Res Synth Methods 2012;3:98-110.

49. Dias S, Welton NJ, Caldwell DM, et al. Checking consistency in mixed treatment comparison meta-analysis. Stat Med 2010;29:932-44.

50. Lu G, Ades AE. Assessing evidence inconsistency in mixed treatment comparisons. J Am Stat Assoc 2006;101:447-59.

51. Veroniki AA, Vasiliadis HS, Higgins JP, et al. Evaluation of inconsistency in networks of interventions. Int J Epidemiol 2013;42:332-45. 